\title{
EVALUACIÓN FORMATIVA Y COMPARTIDA EN LA ASIGNATURA DE PRACTICUM
}

\author{
Formative and Shared Assessment in "Practicum" subject \\ Avaliação formativa e partilhada na disciplina de "Estágio"
}

\begin{abstract}
Cristina Pascual-Arias (1)
Víctor M. López-Pastor (2)

Miriam Molina Soria (3)

(1) Facultad de Educación de Segovia, Universidad de Valladolid, España. Correo electrónico: cristina.pascual@uva.es

(2) Facultad de Educación de Segovia, Universidad de Valladolid, España. Correo electrónico: vlopez@mpc.uva.es

(3) Facultad de Educación de Segovia, Universidad de Valladolid, España. Correo electrónico: miriam.molina@uva.es
\end{abstract}

\section{Resumen}

Presentamos el desarrollo de un proceso de Evaluación Formativa y Compartida en la asignatura de Practicum II durante el curso 2018/2019, con un grupo reducido de 6 alumnas que realizan sus prácticas en aulas de Educación Infantil y Educación Primaria. Hemos desarrollado y utilizado unos instrumentos de EFyC acordes a las actividades de aprendizaje que desarrolla el alumnado de esta asignatura. Plasmamos al final de este texto los primeros resultados encontrados, que completaremos en la presentación de la comunicación durante el Congreso el próximo mes de julio. Como conclusión podemos avanzar que este sistema nos ha permitido desarrollar una evaluación orientada hacia el aprendizaje a lo largo del todo el proceso de prácticas.

Palabras clave: Evaluación formativa; evaluación compartida; prácticum; formación inicial del profesorado

\begin{abstract}
This paper presents the development of a Formative and Shared Assessment process in the subject of "Practicum" during the academic year of 2018/2019 with a small group of 6 students who develop their practices in the early Childhood and Primary Education. We have developed and used Formative and Shared Assessment instruments according to the learning activities developed by students of this subject. The first results were
\end{abstract}


presented at the end of this paper which will be completed during the communication on the Congress in July. As a conclusion we can advance that this system allowed to develop an evaluation oriented toward learning throughout the whole process of practices.

Keywords: Formative assessment; shared assessment; practicum; pre-service teacher education

\section{Resumo}

Neste trabalho apresentamos o desenvolvimento de um processo de Avaliação Formativa e partilhada na disciplina "Estágio" durante o ano letivo de 2018/2019, com um pequeno grupo de 6 alunos que realizam o seu Estágio em aulas de Educação Préescolar e Educação Primária. Desenvolvemos e utilizamos instrumentos de avaliação formativa e partilhada, de acordo com as atividades de aprendizagem desenvolvidas pelos alunos nesta disciplina. Os primeiros resultados obtidos foram apresentados na parte final do texto, sendo os restantes exibidos em julho durante a comunicação no Congresso. Como conclusão, podemos afirmar que este sistema nos permitiu desenvolver uma avaliação orientada para a aprendizagem ao longo de todo o processo de práticas.

Palavras-chave: Avaliação formativa; avaliação partilhada; estágio; formação inicial de professores

\section{Introducción}

Hemos desarrollado nuestra experiencia de Evaluación Formativa y Compartida (EFyC) en la asignatura de Prácticum II. Dado que es el último periodo de prácticas y resulta una de las asignaturas más determinantes. La asignatura de Prácticum II es obligatoria y se compone de un total de 24 ECTS (14,1 ECTS presenciales y 9,9 ECTS no presenciales). En ella se propone: (a) fomentar la observación sistemática y la reflexión; (b) aprender del seguimiento y el asesoramiento del maestro tutor del colegio y del tutor de la Facultad de Educación; (c) desarrollar soluciones para las situaciones prácticas que se encuentran en el colegio uniéndonos con los contenidos teóricos y las competencias que han adquirido. 


\section{Contextualización}

Hemos desarrollado esta experiencia con un grupo de 6 alumnas en la Facultad de Educación de Segovia (Universidad de Valladolid) en la asignatura "Practicum II", 4 ellas cursan $4^{\circ}$ de Grado de Educación Infantil y 2 de ellas $4^{\circ}$ y $5^{\circ}$ del Doble Grado en Educación Infantil y Primaria, respectivamente. Debido al carácter presencial de esta asignatura todos siguen la misma vía de evaluación: la vía continua. También realizan las mismas actividades de aprendizaje, que detallaremos en la tabla 1.

\section{Diseño y desarrollo de la experiencia}

Al ser la primera vez que impartimos la docencia en esta asignatura planteamos un nuevo proceso de EFyC. Hemos diseñado y elaborado documentos de evaluación acordes a las actividades de aprendizaje de esta asignatura, atendiendo a los criterios que proponen López y Pérez (2017). El trabajo del alumnado en los colegios tiene una duración de 12 semanas lectivas, en las que realizan las actividades de aprendizaje recogidas en la tabla 1, en la que presentamos una pequeña descripción de estas y el instrumento de evaluación diseñado y utilizado. La tutorización de las 6 alumnas se coordina con los tutores de los colegios, a través de visitas a los centros y comunicación por correo electrónico.

Tabla 1.

Actividades de aprendizaje durante el Prácticum II y sus instrumentos de EFyC

\begin{tabular}{|c|c|c|}
\hline $\begin{array}{l}\text { Actividad de } \\
\text { aprendizaje }\end{array}$ & Descripción & $\begin{array}{c}\text { Instrumento de } \\
\text { Evaluación }\end{array}$ \\
\hline $\begin{array}{l}\text { Diario selectivo } \\
\text { de carácter } \\
\text { semanal }\end{array}$ & $\begin{array}{l}\text { En este modelo de diario los alumnos en función de sus } \\
\text { vivencias reflexionan cada semana sobre un tema concreto, que } \\
\text { relacionan con sus percepciones, legislación, aportaciones de } \\
\text { autores, etc. Se busca la reflexión sobre aspectos significativos } \\
\text { para su formación y docencia como futuros maestros }\end{array}$ & $\begin{array}{l}\text { Rúbrica de } \\
\text { autoevaluación } \\
\text { del diario }\end{array}$ \\
\hline $\begin{array}{l}\text { Memoria de } \\
\text { practicas }\end{array}$ & $\begin{array}{l}\text { Constituye la elaboración de un documento que refleje las } \\
\text { características del centro donde realiza las prácticas y su } \\
\text { actuación, así como una valoración personal del proceso. Todo } \\
\text { ello realizando un análisis personal y reflexivo de la experiencia } \\
\text { realizada en el Prácticum. }\end{array}$ & $\begin{array}{l}\text { Ficha de } \\
\text { autoevaluación } \\
\text { de la memoria }\end{array}$ \\
\hline $\begin{array}{l}\text { Seminarios } \\
\text { grupales }\end{array}$ & $\begin{array}{l}\text { Durante este periodo los alumnos se reúnen con su tutor de } \\
\text { prácticas en la Facultad de Educación para solventar las dudas } \\
\text { que encuentran, compartir experiencias y reflexionar junto a sus } \\
\text { compañeros. El objetivo del seminario es ayudar y asesorar } \\
\text { sobre cuestiones que surjan en el desarrollo de sus prácticas. }\end{array}$ & $\begin{array}{l}\text { Escala } \\
\text { Graduada: } \\
\text { criterios de } \\
\text { evaluación y } \\
\text { calificación en }\end{array}$ \\
\hline $\begin{array}{l}\text { Desarrollo de las } \\
\text { prácticas en los } \\
\text { Centros Escolares }\end{array}$ & $\begin{array}{l}\text { Las prácticas constituyen un proceso único y progresivo. El } \\
\text { alumnado participará en las tareas de clase, de planificación, } \\
\text { realización y evaluación conjunta y reflexiva con el maestro } \\
\text { tutor del colegio. De esta manera aprenderá a desarrollar su } \\
\text { propia docencia de una manera guiada en un contexto real. }\end{array}$ & $\begin{array}{l}\text { las actividades } \\
\text { del aprendizaje } \\
\text { del Prácticum II. }\end{array}$ \\
\hline
\end{tabular}




\section{Sistema de evaluación utilizado}

En los anexos I, II y III, mostramos los instrumentos que facilitan el proceso de EFyC. Todos los alumnos siguen la vía de evaluación continua, la única posible, pues se requiere la asistencia al $100 \%$ de las horas lectivas de los colegios durante este periodo (con un máximo de un 10\% de faltas justificadas). Estos instrumentos de evaluación nos permiten tener un seguimiento del proceso de prácticum de nuestras alumnas. Así mismo, a ellas les permite mejorar y aprender del feedback que se produce entre profesor-alumno en todo el proceso. También, permiten establecer una evaluación continua, razonada y justificada conjuntamente con las maestras tutoras de los colegios. Todas las actividades de aprendizaje tienen que estar realizadas para superar el proceso de prácticas y todas tienen su peso en la evaluación que se ve reflejado en la escala graduada que se puede encontrar en el anexo III.

\section{Resultados}

A falta de los resultados definitivos que obtendremos al final del proceso, podemos señalar algunos de los primeros resultados, las cuales complementaremos en el congreso del próximo mes de Julio:

- La primera valoración que hacemos del proceso es muy positiva. Utilizar una misma EFyC con criterios establecidos desde el principio nos aporta mucha objetividad, independientemente del contexto o tutor responsable de los alumnos de Prácticum.

- Consideramos que es una experiencia innovadora de EFyC porque supone una gran diferencia con modelos más tradicionales de evaluación de esta asignatura, en los que los alumnos no obtienen valoraciones ni feedback sobre su aprendizaje en todo el proceso; de esta manera pueden reconducir su acción docente en un contexto real.

- Las alumnas están respondiendo muy bien a la implantación de este sistema de EFyC y están aprendiendo del desarrollo de la EFyC como docentes. En cada entrega obtienen un feedback de su propia autoevaluación y comprueban cuales son los aspectos positivos y los que necesitan mejorar, logrando un aprendizaje de su propia práctica docente.

\section{Conclusiones}

Podemos afirmar que los objetivos de nuestro trabajo se están cumpliendo. El sistema de EFyC ha permitido orientar el proceso de Prácticum hacia el aprendizaje del 
alumnado, logrando un mismo proceso de evaluación para todos los alumnos, independientemente de su contexto de prácticas (Martínez-Mínguez, 2017).

Este trabajo puede resultar de interés para los docentes que tutorizan procesos de Prácticum, para profesores universitarios que estén desarrollando prácticas de EFyC o bien que quieran iniciarse en esta temática.

Como prospectiva de trabajo compararemos los resultados que obtengamos este curso con los cursos sucesivos. También puede transferirse este sistema a otras experiencias similares tanto en la misma Universidad como en otras adaptando los instrumentos a las actividades de aprendizaje o contextos.

\section{Referencias}

Martínez-Mínguez, L. (2017). Coevaluación y Heteroevaluación del Practicum a través de una escala de puntuación en la formación inicial del profesorado. En V. M., López-Pastor y A., Pérez-Pueyo (Coords.), Buenas prácticas docentes. Evaluación formativa y compartida en educación: Experiencias de éxito en todas las etapas educativas (pp.422-428). León: Universidad de León.

López, V. M., y Pérez, A. (2017). Introducción a la creación y uso de escalas descriptivas y rúbricas. En V. M., López-Pastor y A., Pérez-Pueyo (Coords.), Buenas prácticas docentes. Evaluación formativa y compartida en educación: Experiencias de éxito en todas las etapas educativas (pp. 92-115). León: Universidad de León. 
Anexo I. Rúbrica de autoevaluación del Diario Selectivo - Prácticum II - 2018-2019

\begin{tabular}{|c|c|c|c|}
\hline \multicolumn{4}{|l|}{ Nombre y Apellidos: } \\
\hline \multicolumn{4}{|l|}{ Fecha y número de entrega del diario: } \\
\hline Nivel A & Nivel B & Nivel C & Nivel D \\
\hline $\begin{array}{l}\text { 1.Muy buena selección de situaciones } \\
\text { vistas o vividas relacionadas con los } \\
\text { temas de la enseñanza. }\end{array}$ & $\begin{array}{l}1 \text { Buena selección de situaciones vistas o } \\
\text { vividas relacionadas con los temas de la } \\
\text { enseñanza. }\end{array}$ & $\begin{array}{l}\text { 1.Mala selección de situaciones vistas } \\
\text { o vividas relacionadas con los temas } \\
\text { de la enseñanza. }\end{array}$ & $\begin{array}{l}\text { 1.Muy mala selección de situaciones } \\
\text { vistas o vividas relacionadas con los } \\
\text { temas de la enseñanza. }\end{array}$ \\
\hline $\begin{array}{l}\text { 2. Muy buena calidad de análisis de las } \\
\text { situaciones elegidas, los aspectos } \\
\text { didácticos y de las competencias } \\
\text { docentes; interesante valoración global. }\end{array}$ & $\begin{array}{l}\text { 2. Buena calidad de análisis de las } \\
\text { situaciones elegidas, los aspectos } \\
\text { didácticos y de las competencias } \\
\text { docentes, incluye valoración global con } \\
\text { algo de reflexión. }\end{array}$ & $\begin{array}{l}\text { 2. Análisis muy simples de las } \\
\text { situaciones es elegidas, los aspectos } \\
\text { didácticos y de las competencias } \\
\text { docentes, poca o escasa valoración } \\
\text { global. }\end{array}$ & $\begin{array}{l}\text { 2. Análisis erróneos de las situaciones } \\
\text { elegidas, los aspectos didácticos y de } \\
\text { las competencias docentes, valoración } \\
\text { global nula y escasa o nula reflexión. }\end{array}$ \\
\hline $\begin{array}{l}\text { 3. Muy buena presentación y estructura } \\
\text { correcta. Redacción adecuada con } \\
\text { sintaxis correcta y sin faltas de } \\
\text { ortografía. }\end{array}$ & $\begin{array}{l}\text { 3. Buena presentación y estructura } \\
\text { correcta. Redacción adecuada con } \\
\text { sintaxis correcta y alguna falta de } \\
\text { ortografía. }\end{array}$ & $\begin{array}{l}\text { 4. Mala presentación y estructura } \\
\text { incorrecta. Mala redacción con } \\
\text { sintaxis inadecuada y con faltas de } \\
\text { ortografía. }\end{array}$ & $\begin{array}{l}\text { 4. Muy mala presentación y estructura } \\
\text { incorrecta o ilegible. Mala redacción } \\
\text { con sintaxis inadecuada y muchas } \\
\text { faltas de ortografía. }\end{array}$ \\
\hline $\begin{array}{l}\text { 4. Muy buena presentación de ideas, } \\
\text { relacionadas entre sí con aportaciones } \\
\text { vividas y otros párrafos o argumentos } \\
\text { presentados. }\end{array}$ & $\begin{array}{l}\text { 4. Buena presentación de ideas, } \\
\text { relacionadas entre sí con aportaciones } \\
\text { vividas y otros párrafos o argumentos } \\
\text { presentados. }\end{array}$ & $\begin{array}{l}\text { 4. Presentación de ideas inconexas } \\
\text { entre sí con aportaciones muy } \\
\text { superficiales y argumentos con poco } \\
\text { sentido. }\end{array}$ & $\begin{array}{l}\text { 4. Muy mala presentación de ideas, } \\
\text { sin ningún tipo de relación entre sí, } \\
\text { sin unión con las aportaciones vividas } \\
\text { y sin argumentos. }\end{array}$ \\
\hline $\begin{array}{l}\text { 5. Muy buen uso de citas de otros } \\
\text { autores y uso correcto del sistema de } \\
\text { citación según la normativa APA. }\end{array}$ & $\begin{array}{l}\text { 5. Buen uso de citas de otros autores y } \\
\text { uso correcto del sistema de citación según } \\
\text { la normativa APA, con algún error. }\end{array}$ & $\begin{array}{l}\text { 5. Escaso uso de citas de otros } \\
\text { autores, con muchos fallos en la } \\
\text { utilización de la normativa APA. }\end{array}$ & $\begin{array}{l}\text { 5. No hay uso de normativa APA ni } \\
\text { uso de citas de otros autores o todo lo } \\
\text { que está citado está incorrecto. }\end{array}$ \\
\hline
\end{tabular}


Anexo II. Ficha de autoevaluación Memoria de Prácticas -Prácticum II 2018-2019

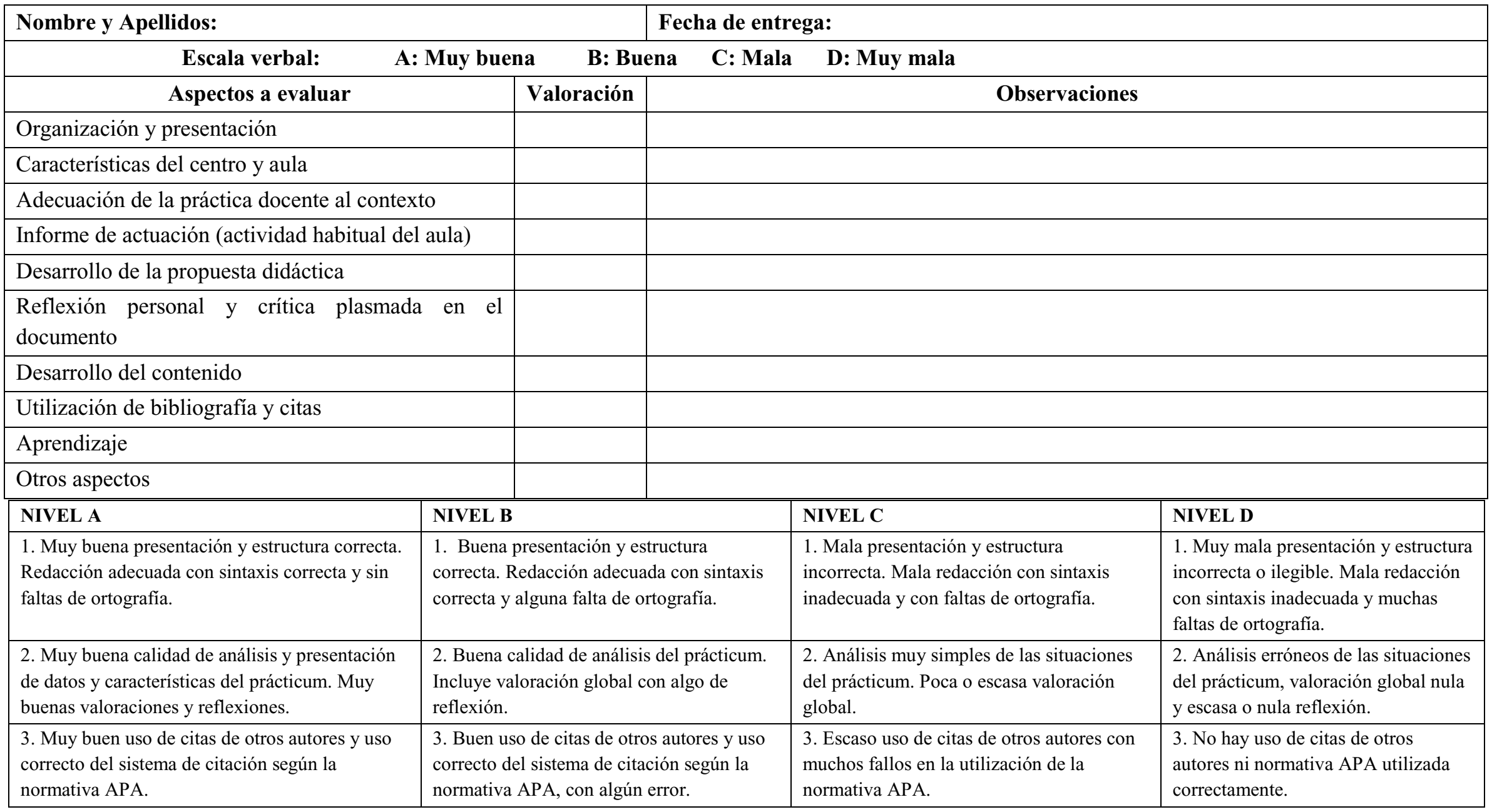


Anexo III. Escala Graduada: Criterios de evaluación y calificación en las actividades de aprendizaje del Prácticum II

\begin{tabular}{|c|c|c|c|c|}
\hline \multicolumn{3}{|c|}{ Nombre y Apellidos: } & \multicolumn{2}{|c|}{ Fecha: } \\
\hline $\begin{array}{l}\text { Actividades de } \\
\text { aprendizaje }\end{array}$ & Nivel A (90-100) & Nivel B (70-89) & Nivel C (50-69) & Nivel D (30-49) \\
\hline $\begin{array}{l}\text { Valoración del } \\
\text { maestro durante } \\
\text { el periodo de } \\
\text { prácticas }\end{array}$ & $\begin{array}{l}\text { 1-Muy buena preparación del } \\
\text { trabajo escolar. } \\
\text { 2- Muy buena realización del } \\
\text { trabajo escolar, con alta } \\
\text { implicación, interacción y } \\
\text { capacidad de respuesta educativa. } \\
\text { 3-Muy buena actitud y cualidades } \\
\text { personales en el desarrollo del } \\
\text { prácticum. }\end{array}$ & $\begin{array}{l}\text { 1- Buena preparación del trabajo escolar. } \\
\text { 2- Buena realización del trabajo escolar, } \\
\text { con alta implicación, interacción y } \\
\text { capacidad de respuesta educativa. } \\
\text { 3-Buena actitud y cualidades personales } \\
\text { en el desarrollo del prácticum. }\end{array}$ & $\begin{array}{l}\text { 1- Mala preparación del trabajo } \\
\text { escolar. } \\
\text { 2- Realización del trabajo escolar } \\
\text { sin apenas implicación, interacción } \\
\text { ni capacidad de respuesta } \\
\text { educativa. } \\
\text { 3-Mala actitud, no muestra } \\
\text { cualidades personales buenas en el } \\
\text { desarrollo del prácticum }\end{array}$ & $\begin{array}{l}\text { 1- Nula preparación del trabajo escolar. } \\
\text { 2- Muy mala realización del trabajo } \\
\text { escolar sin implicación, interacción ni } \\
\text { capacidad de } \\
\text { respuesta educativa. } \\
\text { 3-Muy mala actitud, no muestra } \\
\text { cualidades personales buenas en el } \\
\text { desarrollo del prácticum }\end{array}$ \\
\hline $\begin{array}{l}\text { Elaboración de } \\
\text { documentos: } \\
\text { Diario selectivo } \\
\text { del prácticum + } \\
\text { Memoria de } \\
\text { prácticas }\end{array}$ & $\begin{array}{l}\text { 1-Muy buena presentación, } \\
\text { estructura correcta y claramente } \\
\text { observable. } \\
\text { 2-Muy buena calidad de análisis de } \\
\text { los aspectos del prácticum, muy } \\
\text { interesante valoración global y } \\
\text { reflexión crítica. } \\
\text { 3-Muy buena selección de } \\
\text { situaciones vistas o vividas } \\
\text { relacionadas con los temas de la } \\
\text { enseñanza. } \\
\text { 4- Muy buen uso de citas de otros } \\
\text { autores y uso correcto del sistema } \\
\text { de citación según la normativa } \\
\text { APA. }\end{array}$ & $\begin{array}{l}\text { 1- Buena presentación, estructura correcta } \\
\text { y claramente observable. } \\
\text { 2- Buena calidad de análisis de los } \\
\text { aspectos del prácticum, con buenas } \\
\text { valoraciones y reflexiones críticas. } \\
\text { 3- Buena selección de situaciones vistas o } \\
\text { vividas relacionadas con los temas de la } \\
\text { enseñanza. } \\
\text { 4- Buen uso de citas de otros autores y } \\
\text { uso correcto del sistema de citación según } \\
\text { la normativa APA con algún error. }\end{array}$ & $\begin{array}{l}\text { 1- Mala presentación y estructura } \\
\text { aceptable. } \\
\text { 2- Mala calidad de análisis de los } \\
\text { aspectos del prácticum, con pocas } \\
\text { valoraciones y reflexiones críticas. } \\
\text { 3- Mala selección de situaciones } \\
\text { vistas o vividas relacionadas con } \\
\text { los temas de la enseñanza. } \\
\text { 4- Uso incorrecto de citas de otros } \\
\text { autores y del sistema de citación } \\
\text { según la normativa APA con } \\
\text { errores. }\end{array}$ & $\begin{array}{l}\text { 1- Muy mala presentación y estructura } \\
\text { incorrecta. } \\
\text { 2- Muy mala calidad de análisis de los } \\
\text { aspectos del prácticum, aspectos } \\
\text { inconexos, sin valoraciones y reflexiones } \\
\text { críticas. } \\
\text { 3- Muy mala selección de situaciones } \\
\text { vistas o vividas relacionadas con los } \\
\text { temas de la enseñanza. } \\
\text { 4- Uso incorrecto de citas de otros } \\
\text { autores y del sistema de citación según la } \\
\text { normativa APA con muchos errores. }\end{array}$ \\
\hline $\begin{array}{l}\text { Seminarios de } \\
\text { seguimiento }\end{array}$ & $\begin{array}{l}\text { 1-Muy buenas y habituales } \\
\text { intervenciones en los seminarios y } \\
\text { aportaciones a los compañeros. } \\
\text { 2-Asistencia a todos los seminarios }\end{array}$ & $\begin{array}{l}\text { 1- Buenas intervenciones en los } \\
\text { seminarios y aportaciones a los } \\
\text { compañeros. } \\
\text { 2-Asistencia a la mayoría de seminarios }\end{array}$ & $\begin{array}{l}\text { 1-Escasas intervenciones en los } \\
\text { seminarios y pocas aportaciones a } \\
\text { los compañeros. } \\
\text { 2-Asistencia a pocos seminarios }\end{array}$ & $\begin{array}{l}\text { 1-Nulas intervenciones en los seminarios } \\
\text { y muy pocas aportaciones a los } \\
\text { compañeros. } \\
\text { 2-Una o ninguna asistencia a seminarios }\end{array}$ \\
\hline
\end{tabular}

